\title{
Effect of temperature on spiking patterns of neocortical layer $2 / 3$ and layer 6 pyramidal neurons
}

\author{
Tristan Hedrick* and Jack Waters \\ Department of Physiology, Feinberg School of Medicine, Northwestern University, Chicago, IL, USA
}

\section{Edited by:}

Michael Brecht, Humboldt

University Berlin, Germany

Reviewed by:

Johannes J. Letzkus, Friedrich

Miescher Institute for Biomedical

Research, Switzerland

Allan T. Gulledge, Geisel School of

Medicine at Dartmouth, USA

*Correspondence:

Tristan Hedrick, Department of Physiology, Feinberg School of

Medicine, Northwestern University,

303 E. Chicago Ave., Chicago,

IL 60611, USA.

e-mail: tristanhedrick2008@

u.northwestern.edu
The spiking patterns of neocortical pyramidal neurons are shaped by the conductances in their apical dendrites. We have previously shown that the spiking patterns of layer 5 pyramidal neurons change with temperature, probably because temperature modulates the electrical coupling between somatic and dendritic compartments. Here we determine whether temperature has similar effects on the spiking patterns of layer $2 / 3$ and layer 6 pyramidal neurons in acute slices of mouse primary motor cortex. In both cell types, decreasing temperature led to more irregular spiking patterns. Our results indicate that a decrease in spiking regularity with decreasing temperature, probably mediated by increased electrical coupling between soma and dendrites, is common to all pyramidal neurons in motor cortex.

Keywords: neocortex, dendrite, firing pattern, motor cortex, electrical coupling

\section{INTRODUCTION}

The spiking patterns of pyramidal neurons in layers $2 / 3$ and 6 of the neocortex are generally described as regular, meaning that spikes are evenly spaced during constant current injection (Connors et al., 1982; McCormick et al., 1985; Agmon and Connors, 1989; Chagnac-Amitai et al., 1990; Connors and Gutnick, 1990; Nuñez et al., 1993; Cho et al., 2004). However, differences in inter-spike interval (ISI) variability and spike frequency adaptation have been reported among regularly spiking pyramidal neurons and it is unclear whether these differences reflect neuron-to-neuron variablity or result from the different recording conditions employed in different studies (Agmon and Connors, 1992; Nuñez et al., 1993; Dégenètais et al., 2002; Cho et al., 2004).

In contrast with layer $2 / 3$ and layer 6 pyramidal neurons, layer 5 pyramidal neurons have been classified as intrinsically bursting or regularly firing (Connors et al., 1982; McCormick et al., 1985; Agmon and Connors, 1989; Chagnac-Amitai et al., 1990; Mason and Larkman, 1990). Bursting is associated with the regenerative activation of sodium and calcium currents in the apical dendrite that cause prolonged depolarization of the axon initial segment (Silva et al., 1991; Schwindt and Crill, 1999; Williams and Stuart, 1999; Larkum et al., 2001; Larkum and Zhu, 2002).

Although pyramidal neurons in layer $2 / 3$ and layer 6 both express voltage-gated sodium and calcium conductances in the apical dendrite and these conductances are capable of supporting dendritic spike initiation, the resulting depolarization is less prolonged than in layer 5 pyramidal neurons. As a result depolarization of the axon initial segment is relatively brief and does not cause a burst (Waters et al., 2003; Larkum et al., 2007; Ledergerber and Larkum, 2010).
We have previously reported that the spiking patterns of layer 5 pyramidal neurons are sensitive to temperature. Layer 5 neurons spike in bursts at room temperature, but adopt a regular firing pattern at physiological temperatures (Hedrick and Waters, 2011). This change in spiking pattern may result from greater electrical coupling of soma and dendrite at lower than physiological temperatures and may help explain differences between the spiking patterns reported in different studies.

Current injection into the apical dendrites of layer 6 pyramidal neurons evokes spiking which is more irregular than during somatic current injection, the ISI being more variable during dendritic current injection (Ledergerber and Larkum, 2010). Hence if electrical coupling between soma and apical dendrite increases with decreasing temperature, we might expect the spiking patterns of layer $2 / 3$ and layer 6 pyramidal neurons to be less regular at lower temperatures. To test this hypothesis we determined the effects of temperature on the spiking patterns of layer $2 / 3$ and layer 6 pyramidal neurons. We show that the spiking patterns of layer $2 / 3$ and layer 6 pyramidal neurons become less regular when the temperature is decreased below physiological temperatures, as in layer 5 pyramidal neurons. Hence pyramidal neurons throughout neocortex spike with decreasing regularity as temperature decreases below the physiological range, with the precise pattern of spiking at low temperatures presumably being determined largely by dendritic conductances.

\section{MATERIALS AND METHODS}

All experiments and procedures were approved by the Northwestern University Institutional Animal Care and Use Committee (IACUC). 


\section{SLICE PREPARATION}

P42-P54 C57BL-6 mice were anaesthetized using an interperitoneal injection of $120 \mathrm{mg} / \mathrm{kg}$ ketamine and $50 \mathrm{mg} / \mathrm{kg}$ xylazine in phosphate-buffered saline (PBS): $75 \mathrm{mM} \mathrm{Na}_{2} \mathrm{HPO}_{4}, 25 \mathrm{mM}$ $\mathrm{NaH}_{2} \mathrm{PO}_{4}$, pH 7.4, transcardially perfused with ice-cold artificial cerebrospinal fluid (ACSF): $125 \mathrm{mM} \mathrm{NaCl}, 2.5 \mathrm{mM} \mathrm{KCl}, 1.25 \mathrm{mM}$ $\mathrm{NaH}_{2} \mathrm{PO}_{4}, 20 \mathrm{mM} \mathrm{NaHCO}, 5 \mathrm{mM}$ HEPES, $25 \mathrm{mM}$ glucose, $1 \mathrm{mM} \mathrm{CaCl}_{2}, 2 \mathrm{mM} \mathrm{MgCl}_{2}, \mathrm{pH} 7.3$, gassed with $95 \% \mathrm{O}_{2} / 5 \% \mathrm{CO}_{2}$, and decapitated. The brain was quickly removed into ice-cold ACSF. Three hundred micrometer-thick parasagittal slices were cut using a vibrating microslicer (Vibratome, St. Louis, MO). Slices were held in ACSF at $37^{\circ} \mathrm{C}$ for $5-15 \mathrm{~min}$ and thereafter at room temperature until use.

\section{ELECTROPHYSIOLOGY}

Slices were transferred to the stage of an upright microscope (Olympus BX51) and continuously perfused with ACSF gassed with $95 \% \mathrm{O}_{2} / 5 \% \mathrm{CO}_{2}$ and warmed to $35-37^{\circ} \mathrm{C}$. Whole-cell recordings were obtained from pyramidal neurons in layers $2 / 3$ and 6 of primary motor cortex under visual guidance with a $\times 20$ /NA 1.0 water-immersion objective and infra-red differential interference contrast optics. Layer $2 / 3$ pyramidal neurons were identified by their somatic shape, the location of their somata $150-500 \mu \mathrm{m}$ from the pial edge of the slice, and their large apical dendrites that were visible by fluorescence microscopy once filled with indicator. Layer 6 pyramidal neurons were likewise visually identified, $1300-1600 \mu \mathrm{m}$ from the pial edge of the slice. Whole-cell recording pipettes were 5-9 M $\Omega$ when filled with intracellular solution: $135 \mathrm{mM} \mathrm{K}$ gluconate, $4 \mathrm{mM} \mathrm{KCl}, 10 \mathrm{mM}$ HEPES, $10 \mathrm{mM} \mathrm{Na}$-phosphocreatine, $4 \mathrm{mM} \mathrm{Mg-ATP,} 0.3 \mathrm{mM}$ $\mathrm{Na}_{2}-\mathrm{GTP}, 0.2 \%$ (w/v) biocytin, $10 \mu \mathrm{M}$ Alexa 594, pH 7.3. Signals were recorded at $50 \mathrm{kHz}$ with an Axoclamp-2A amplifier in Bridge mode (Molecular Devices, Sunnyvale, CA), National Instruments A-to-D boards and Labview software written by Jack Waters (National Instruments, Austin, TX). After recording the morphology of the neuron was routinely checked by fluorescence microscopy to verify that the apical dendritic tree was largely intact.

\section{MEASUREMENT AND CONTROL OF TEMPERATURE}

ACSF was warmed with a thermistor-based in-line solution heater, placed a few inches from the slice. The temperature of the solution was monitored upon entry to the perfusion chamber, a few millimeters from the slice, and maintained at $35-37^{\circ} \mathrm{C}$ by a feedback circuit and temperature controller (TC-324B; Warner Instruments, Hamden, CT). Flow rate was $\sim 3-6 \mathrm{ml} / \mathrm{min}$. The temperature of the ACSF under the objective was verified with a BAT-12 Microprobe thermometer (Physitemp Instruments, Clifton, NJ), using a small 24-gauge polyurethane coated wire probe with polyester insulated thermocouple bead (IT-24P; maximum tip diameter $230 \mu \mathrm{m}$ ), which was placed under the microscope objective. The temperature of ACSF under the objective, measured using the IT-24P probe, was consistently no more than $0.5^{\circ} \mathrm{C}$ cooler than the temperature of the ACSF entering the perfusion chamber, measured by the TC-324B temperature controller.

\section{ANALYSIS}

Analysis was performed using routines written by Jack Waters in IgorPro v6.0 (Wavemetrics, Lake Oswego, OR). Spiking patterns were assessed from $600 \mathrm{~ms}$ current injections at the soma which resulted in 10-12 spikes. We calculated ISIs for each pair of spikes, from spikes 1 and 2 (ISI 1) to spikes 9 and 10 (ISI 9). Spiking patterns were defined as regular, weak bursting, adapting, and strong bursting based on two measures: the similarity of ISIs 4-9, and the ratio of the last/firstISI (ISI 9/ISI 1). The four spiking patterns were defined as follows:

- Strong burst: ISIs 4-9 all differed from the mean of ISIs 4-9 by more than $10 \%$.

- Adapting: some, but not all, of ISIs 4-9 differed from the mean of ISIs $4-9$ by more than $10 \%$.

- Weak burst: none of ISIs 4-9 differed from the mean of ISIs 4-9 by more than $10 \%$ and the ratio of ISIs 9 and 1 (ISI 9/ISI 1) was 2 or more.

- Regular: none of ISIs 4-9 differed from the mean of ISIs 4-9 by more than $10 \%$ and the ratio of ISIs 9 and 1 (ISI 9/ISI 1) was less than 2 .

To compare the regularity of spiking across populations of neurons (Figure 2D), we calculated a regularity index. We gave each spiking pattern a score, which was greater for more regular spiking patterns. Hence a strong bursting pattern was scored as 1 , an adapting pattern as 2 , a weak bursting pattern as 3 , and a regular spiking pattern as 4 . After scoring each neuron at both physiological and cooler temperatures, we calculated the mean score for layer $2 / 3$, layer 5 , and layer 6 pyramidal neurons.

Spiking patterns were calculated from a single representative trial, except where stated otherwise. In most neurons we verified that the spiking pattern in each condition was reproducible in 2-3 trials.

V-I relationships were derived from the steady-state voltage during $600 \mathrm{~ms}$ current steps and were fit with the following relationship (Waters and Helmchen, 2006):

$$
\Delta V=R_{N, 0} \Delta I+C_{A R} \Delta I^{2}
$$

where $R_{N}$ is the resting input resistance (slope at $I=0$ ) and $C_{A R}$ is a quadratic coefficient which describes the curvature of the V-I relationship.

Voltage sag was estimated as the ratio of steady-state and peak voltages during the $600 \mathrm{~ms}$ current step (sag ratio). The membrane time constant was determined by fitting an exponential to the rising phase of the voltage response during the current step.

Action potential threshold was defined as the point at which the first temporal derivative of the voltage $(\mathrm{dV} / \mathrm{dt})$ first exceeded $55 \mathrm{mV} / \mathrm{ms}$. Action potential amplitude was measured from threshold. Action potential half width was measured at half the amplitude. Rheobase was defined as the minimum amount of current required to evoke spiking.

Unless otherwise stated, statistical comparisons were performed using the Wilcoxon matched-pairs signed-ranks test in Graphpad Instat 3.06 (Graphpad Software, La Jolla, CA). 


\section{RESULTS}

\section{CHARACTERIZATION OF SPIKING PATTERNS FOR LAYER 2/3 AND LAYER 6 PYRAMIDAL NEURONS}

We obtained whole-cell recordings from the somata of layer $2 / 3$ and layer 6 pyramidal neurons in primary motor cortex in acute slices from adult mice. Perfusing ACSF was initially maintained at $36-37^{\circ} \mathrm{C}$. Neurons were depolarized by constant somatic current injection for $600 \mathrm{~ms}$, during which neurons fired continuously. Spikes were, to a first approximation, evenly spaced, but many neurons displayed frequency adaptation, with ISIs increasing during the spike train (Figure 1).

\section{A regular spiking}

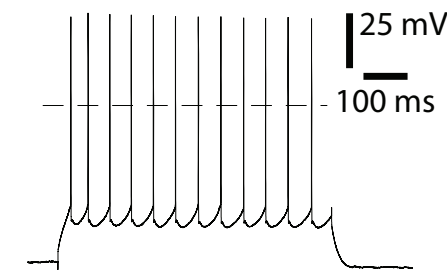

B weak burst
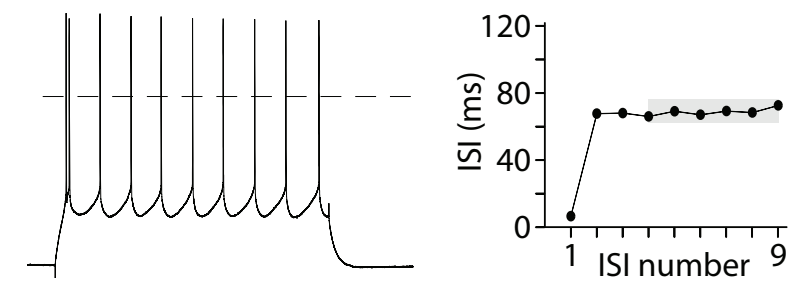

c adapting
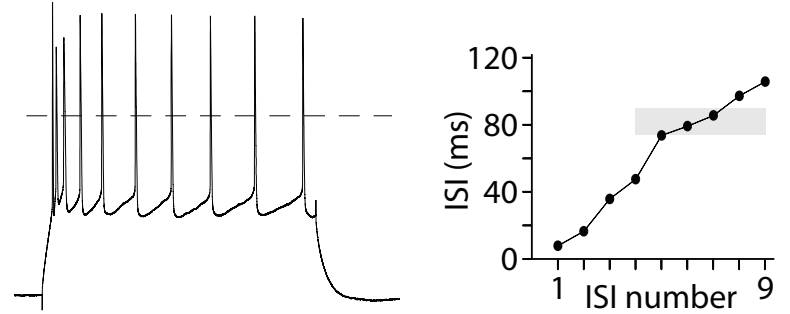

D

strong burst
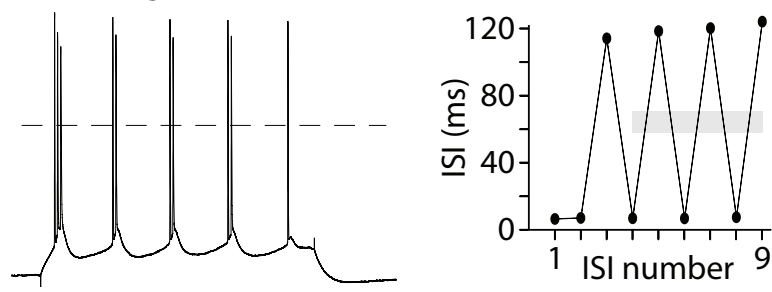

FIGURE 1 | Examples of four spiking patterns of cortical pyramidal neurons. Example spiking patterns and ISIs for each of the four firing patters in our classification system: regular spiking (A), weak bursting (B), adapting (C), strong bursting (D). Spiking was evoked with a $600 \mathrm{~ms}$ constant current injection via the somatic recording pipette. Dashed lines indicate $0 \mathrm{mV}$. Spiking patterns are arranged from most regular (A) to least regular (D). Currents were $300 \mathrm{pA}$ (A and B), $325 \mathrm{pA}$ (C) and $150 \mathrm{pA}$ (D). Gray boxes are drawn from ISI 4 to ISI 9. The upper and lower boundaries of the boxes are at $110 \%$ and $90 \%$ of the mean of ISIs $4-9$.
In an earlier study of layer 5 pyramidal neurons, we classified neurons as bursting or regularly firing based on the variation in ISI during a depolarizing current pulse at the soma, with neurons characterized as regularly spiking if none of the ISIs differed more by than $15 \%$ from the mean ISI and bursting if any ISI differed from the mean by $15 \%$ or more (Hedrick and Waters, 2011). Using this criterion, most layer $2 / 3$ and layer 6 pyramidal neurons would be classified as bursting neurons, despite firing with fairly evenly spaced spikes. Hence we found that this earlier classification failed to adequately describe the spiking patterns of layer $2 / 3$ and layer 6 pyramidal neurons.

Here we adopted a different methodology to classify the spiking patterns, which included two non-bursting patterns: regularly spiking and adapting. Our new classification is based on two measures: the regularity of ISIs 4-9 and the ratio of the last and first ISIs (ninth ISI/first ISI). If all of ISIs 4-9 differed from the mean of ISIs $4-9$ by more than $10 \%$, the spiking pattern was classified as strong bursting. If some but not all of ISIs 4-9 differed from the mean by more than $10 \%$, the spiking pattern was classified as adapting. If none of ISIs 4-9 differed from the mean by more than $10 \%$, neurons were classified as weak bursting or regularly spiking, depending on the ratio of the last and first ISIs: where the ratio of the last and first ISIs was two or more, the pattern was classified as weak bursting; where less than two, it was regularly spiking.

To consider the overall effect of temperature on spiking pattern we ranked the four spiking patterns in order of the regularity of ISIs: from the regularly spiking pattern; to the weakly bursting pattern, which consists of regularly spaced spikes after an initial spike doublet; to the adapting pattern, in which the ISI changes markedly over the course of the spike train; to the strong bursting pattern, in which the ISIs are extremely irregular (Figure 1).

\section{SPIKING PATTERNS OF LAYER 2/3 AND LAYER 6 PYRAMIDAL NEURONS CHANGE WITH TEMPERATURE}

Using this approach, we classified the spiking patterns of layer $2 / 3$ pyramidal neurons. At physiological temperatures $\left(36-37^{\circ} \mathrm{C}\right)$ two layer $2 / 3$ pyramidal neurons displayed regular spiking patterns, six weak bursting patterns, and two adapting patterns (Figure 2A). At $24-26^{\circ} \mathrm{C}$, one neuron displayed a regular spiking pattern and the other nine were adapting (Figure 2A). Hence the regularity of spiking by $\mathrm{L} 2 / 3$ pyramidal neurons decreased with decreasing temperature.

At both temperatures the ISIs increased progressively throughout the spike train in layer $2 / 3$ neurons (for every neuron, the first ISI was smaller than the ninth ISI; $P<0.05$ at both $36-37^{\circ} \mathrm{C}$ and $24-26^{\circ} \mathrm{C}$ ) with this increase being more pronounced at $24-26^{\circ} \mathrm{C}$ than at physiological temperatures (ISI 9/ISI 1 was $3.8 \pm 0.6$ at $24-26^{\circ} \mathrm{C} ; 2.2 \pm 0.2$ at $36-37^{\circ} \mathrm{C}$; $P<0.05)$.

At physiological temperatures $\left(36-37^{\circ} \mathrm{C}\right)$ five layer 6 pyramidal neurons displayed regular, four weak bursting and one adapting spiking patterns (Figure 2C). Upon decreasing the temperature to $24-26^{\circ} \mathrm{C}$, the regularity of ISIs decreased in nine of these 10 neurons, which then adopted weak bursting, adapting or strong bursting patterns (Figure 2C). Only the adapting neuron 

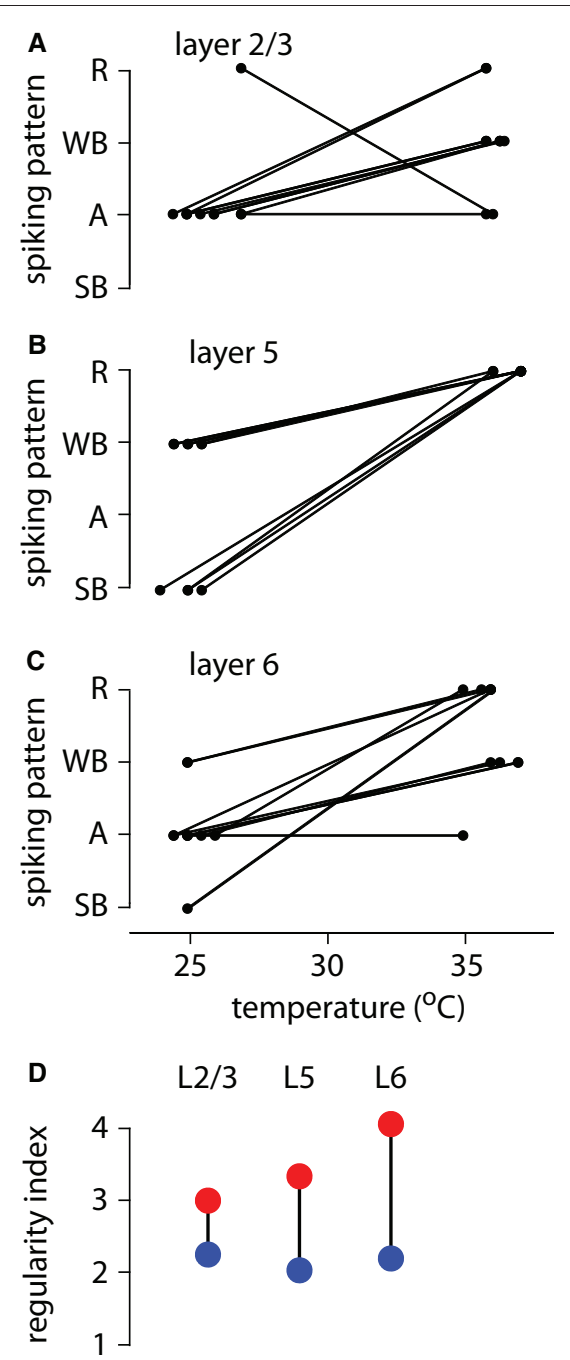

FIGURE 2 | Spiking patterns at low and physiological temperatures. (A) Summary of spiking patterns for 10 layer 2/3 pyramidal neurons at low and high temperatures. All neurons except for one display adapting spiking patterns (A) at low temperatures and regular spiking (R), weak bursting (WB) or adapting (A) at physiological temperatures. (B) Summary of spiking patterns at low and high temperatures for the same 10 layer 5 pyramidal neurons described previously (Hedrick and Waters, 2011), but were re-analyzed using our updated approach to classifying spiking patterns. (C) Summary of spiking patterns for 10 layer 6 pyramidal neurons at low and high temperatures. The majority of neurons were adapting at low temperatures and either weak bursting or regular spiking at physiological temperature. (D) Summary plot showing the decrease in regularity of spiking in layer $2 / 3$, layer 5 , and layer 6 pyramidal neurons with decreasing temperature. Blue dots and red dots represent average index of regularity at room temperature and physiological temperature, respectively.

retained its spiking pattern at physiological temperatures. Hence the regularity of spiking by layer 6 pyramidal neurons decreased with decreasing temperature.

In layer 6, ISIs increased during the spike train (the first ISI was smaller than the ninth ISI; $P<0.05$ at both $36-37^{\circ} \mathrm{C}$ and $24-26^{\circ} \mathrm{C}$ ) with this increase being more pronounced at $24-26^{\circ} \mathrm{C}$ than at physiological temperatures (ISI 9/ISI 1 was $7.5 \pm 1.9$ at $24-26^{\circ} \mathrm{C} ; 5.6 \pm 2.2$ at $\left.36-37^{\circ} \mathrm{C} ; P<0.05\right)$.
The change in spiking pattern is due to temperaturedependent changes in the intrinsic properties of the pyramidal neurons. In five layer 6 pyramidal neurons, we studied the effect of temperature in the presence of glutamate and GABA receptor antagonists $(10 \mu \mathrm{M}$ NBQX, $10 \mu \mathrm{M}$ CPP, $3 \mu \mathrm{M}$ gabazine, and $1 \mu \mathrm{M}$ CGP 52432, applied in the perfusing solution). Spiking pattern changed with temperature in all five neurons, indicating that the change in spiking pattern is not due to temperaturedependent changes in spontaneous synaptic activity.

When we applied this new spiking pattern classification to our previous recordings from layer 5 pyramidal neurons (Hedrick and Waters, 2011), it replicated the results of our previous study, with all layer 5 neurons adopting regular spiking patterns at physiological temperatures and an even split between strong and weak bursting at colder temperatures (Figure 2B). Hence layer 2/3, layer 5 , and layer 6 pyramidal neurons all display more variable ISIs at $24-26^{\circ} \mathrm{C}$ than at physiological temperatures, but with most layer $2 / 3$ and layer 6 neurons adopting adapting spiking patterns at colder temperatures, rather than the bursting patterns observed in layer 5 .

\section{CHANGE IN SPIKING PATTERN IS REVERSIBLE}

In both layer $2 / 3$ and layer 6 spiking patterns were reproducible, with neurons exhibiting the same spiking pattern on repeated trials in each condition (Figures 3A-C). To check on reversibility, we toggled the temperature from physiological temperatures to colder temperatures and back to physiological temperatures, generally 2-3 times (Figures 3A-C). The effects of temperature were reversible: neurons switched back to their original spiking patterns upon rewarming the slice to physiological temperatures and upon cooling and rewarming the slice a second and subsequent time the same changes in spiking pattern were observed (Figures 3A-D).

\section{TEMPERATURE SENSITIVITY OF ACTION POTENTIAL WAVEFORM}

Action potential threshold, amplitude, and half width changed during a spike train in some recordings (Figure 4A). However, across the population of neurons the threshold, amplitude, and half width remained fairly constant during the train for all spiking patterns for both layer $2 / 3$ neurons (Figure 4B) and layer 6 neurons (not shown) at physiological temperatures. Hence to examine changes in action potential waveforms with temperature, we pooled results from neurons with different spiking patterns (Figures 5 and $\mathbf{6}$ ).

In both layer $2 / 3$ and layer 6 neurons, the threshold of the first action potential was similar at physiological temperatures and at $24-26^{\circ} \mathrm{C}$ (each $P>0.05$; Figures 5D and 6D). Threshold changed little during the train at physiological temperatures in layer $2 / 3$ and layer 6 , but shifted to more depolarized potentials at $24-26^{\circ} \mathrm{C}$ (each $P<0.05$; Figures 5D and 6D). Hence in both cell types, threshold was more depolarized for the majority of action potentials at $24-26^{\circ} \mathrm{C}$ than at physiological temperatures (each $P<0.05)$. In contrast, action potential amplitude changed little either during a train or with temperature.

The half width of the first action potential increased upon cooling to $24-26^{\circ} \mathrm{C}$ in layer $2 / 3$ and layer 6 pyramidal neurons (each $P<0.05$; Figures $5 \mathrm{D}$ and $6 \mathrm{D}$ ), and the half width 


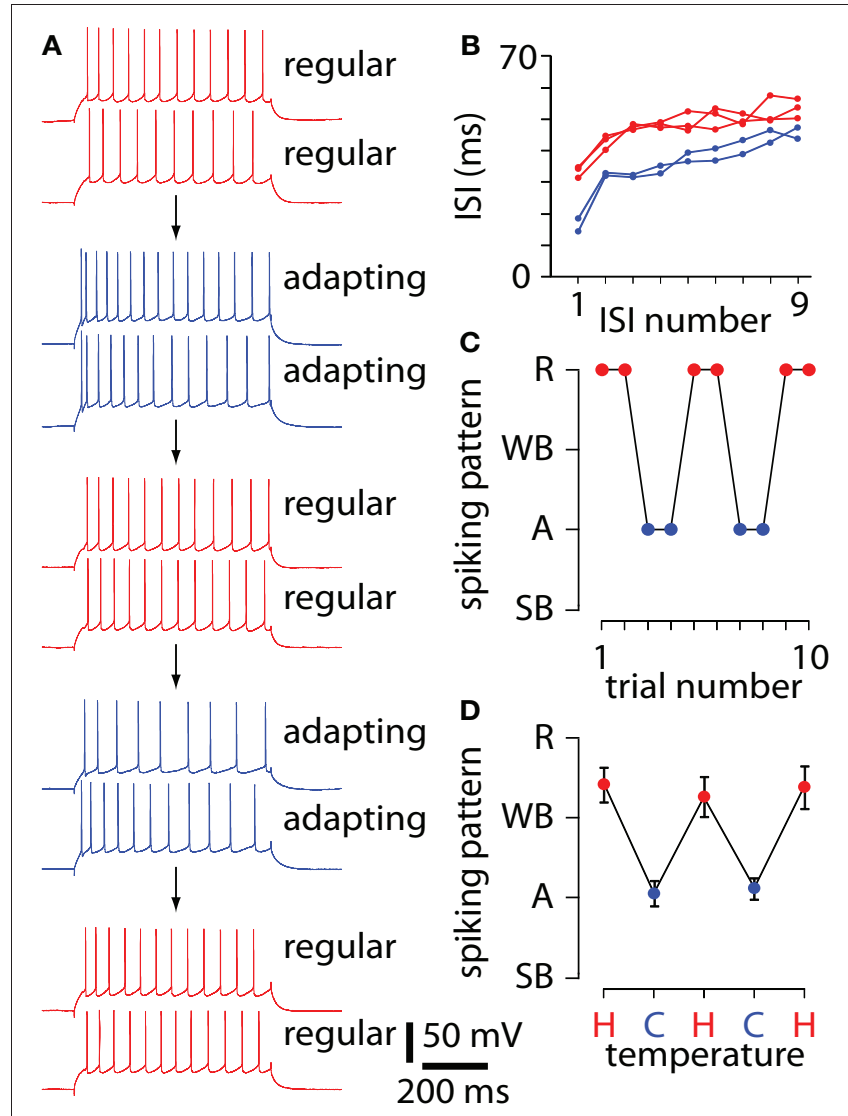

FIGURE 3 | Reversibility of the effect of temperature on spiking pattern. (A) Voltage recordings from a layer 6 pyramidal neuron, showing spiking patterns in a series of trials, during which temperature was repeatedly cycled from $35-37^{\circ} \mathrm{C}$ (red) to $24-26^{\circ} \mathrm{C}$ (blue) and back to $35-37^{\circ} \mathrm{C}$. Trials are displayed in order of acquisition, from top to bottom. Spiking pattern was stable at $24-26^{\circ} \mathrm{C}$ (blue) and at $35-37^{\circ} \mathrm{C}$ (red), but switched between regular and adapting spiking patterns with temperature. (B) The first nine inter-spike intervals (ISIs) for the first of each pair of trials at $24-26^{\circ} \mathrm{C}$ (blue) and at $35-37^{\circ} \mathrm{C}(\mathrm{red})$, illustrating the reproducible and reversible change in spiking pattern. (C) Summary of the changes in spiking pattern for the 10 trials in panel A. (D) Mean \pm SEM spiking patterns for 10 layer 6 pyramidal neurons, showing reproducible changes in spiking pattern with repeated cooling and reheating cycles. Each point is the mean of 3 trials in each neuron, with $n=10,10,9,5$, and 5 neurons contributing to the five points.

of subsequent action potentials in the train increased markedly at $24-26^{\circ} \mathrm{C}$ (each $P<0.05$; Figures 5D and 6D). Hence in both layer $2 / 3$ and layer 6 pyramidal neurons, action potential threshold, and half width change after the initial spikes at low, but not at physiological temperatures.

\section{TEMPERATURE SENSITIVITY OF INTRINSIC MEMBRANE PROPERTIES}

The intrinsic membrane properties of layer $2 / 3$ and layer 6 pyramidal neurons changed with temperature (Figures $5 \mathrm{E}$ and $6 \mathrm{E}$ ). In both layer $2 / 3$ and layer 6 neurons, there was no difference in resting membrane potential at physiological and lower temperatures (each $P>0.05$ ). Both layer $2 / 3$ and layer 6 neurons displayed little membrane sag, consistent with previous reports (Larkum et al., 2007; Ledergerber and Larkum, 2010) and sag was not affected by temperature (each $P>0.05$; Figures 5B,E and 6B,E).

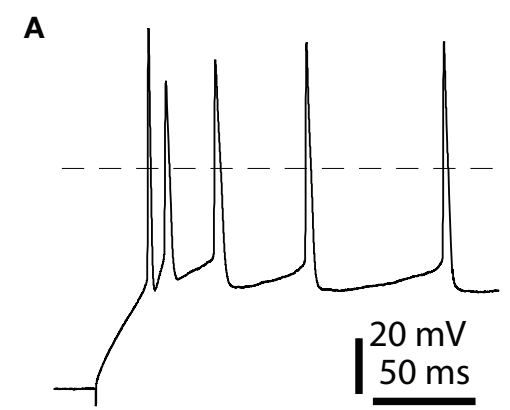

B

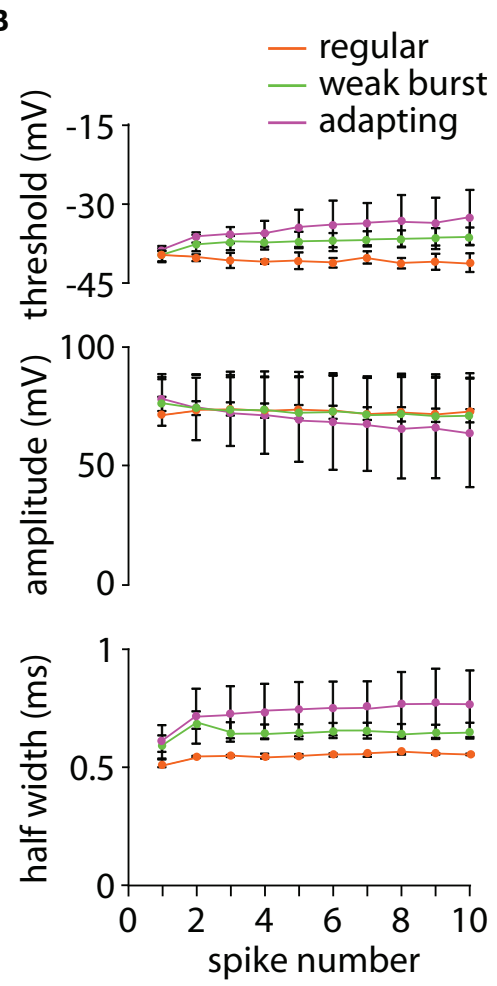

FIGURE 4 | Changes in action potential waveform during a train of spikes. (A) Example of a recording in which spike threshold, amplitude, and half width change during a spike train. Layer 6 neuron with adapting spike pattern at $24-26^{\circ} \mathrm{C}$. (B) Spike threshold, amplitude, and half width for the first 10 spikes during $600 \mathrm{~ms}$ constant current injection at $36-37^{\circ} \mathrm{C}$ in layer $2 / 3$ pyramidal neurons. Neurons were grouped according to spike pattern, with adapting, weak bursting, and regular spiking patterns in pink, green, and orange, respectively. Points represent the mean ( \pm SEM) from two neurons with adapting patterns, six with weak bursting patterns, and two with regular spiking patterns.

Resting input resistance and membrane time constant both approximately doubled at $24-26^{\circ} \mathrm{C}$ in both layers (each $P<$ $0.05)$. At $24-26^{\circ} \mathrm{C}$, in both layer $2 / 3$ and layer 6 neurons membrane rectification was $\sim 2-3$ times greater than at physiological temperatures (each $P<0.05$ ) and rheobase decreased by approximately 25-50\% (each $P<0.05$; Figures 5C,E and 6C,E). These effects are similar to the changes in intrinsic membrane properties we reported for layer 5 pyramidal neurons (Hedrick and Waters, 2011). 

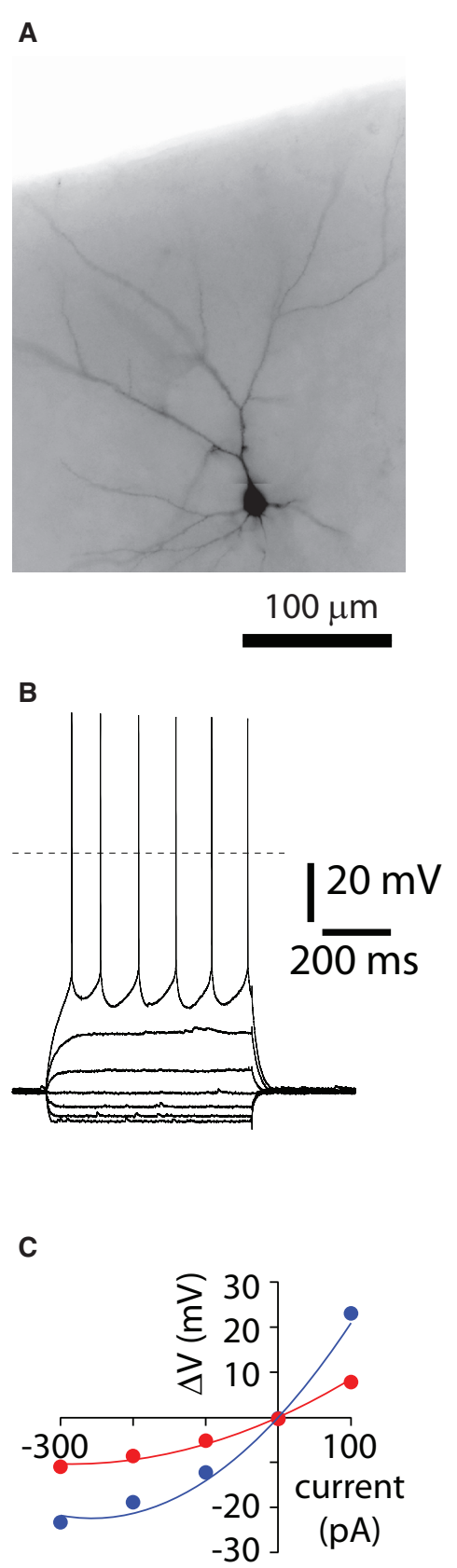

D
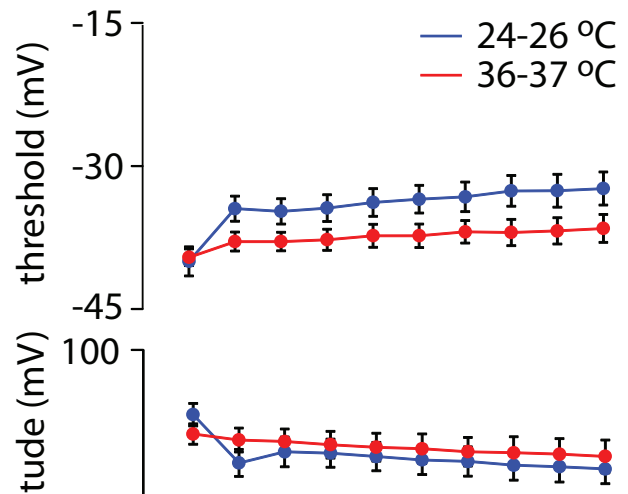

50

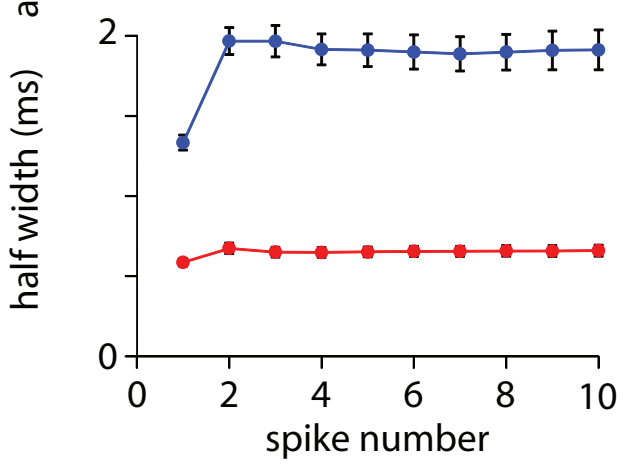

E
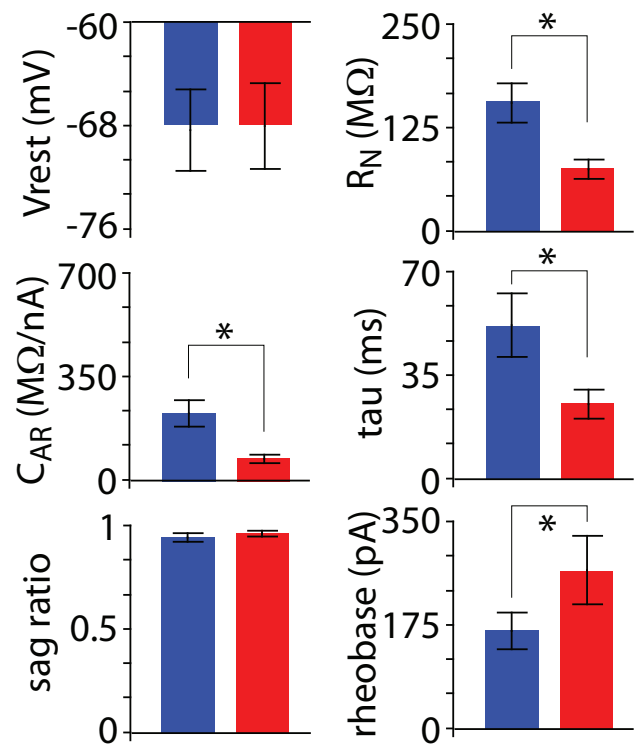

and curves are fits to $\Delta V=R_{N, 0} \Delta I+c_{A R} \Delta I^{2}$ (Waters and Helmchen, 2006). (D) Spike threshold, amplitude, and half width during $600 \mathrm{~ms}$ constant current injection at $36-37^{\circ} \mathrm{C}$ (red) and $24-26^{\circ} \mathrm{C}$ (blue) in 10 layer 2/3 pyramidal neurons. (E) Effects of temperature on resting membrane potential $\left(V_{\text {rest }}\right)$, input resistance $\left(R_{N}\right)$, anomalous rectification $\left(C_{A R}\right)$, membrane time constant (tau), membrane sag, and rheobase. Each bar represents the mean \pm SEM from 10 neurons. * Indicates $P<0.05$. 

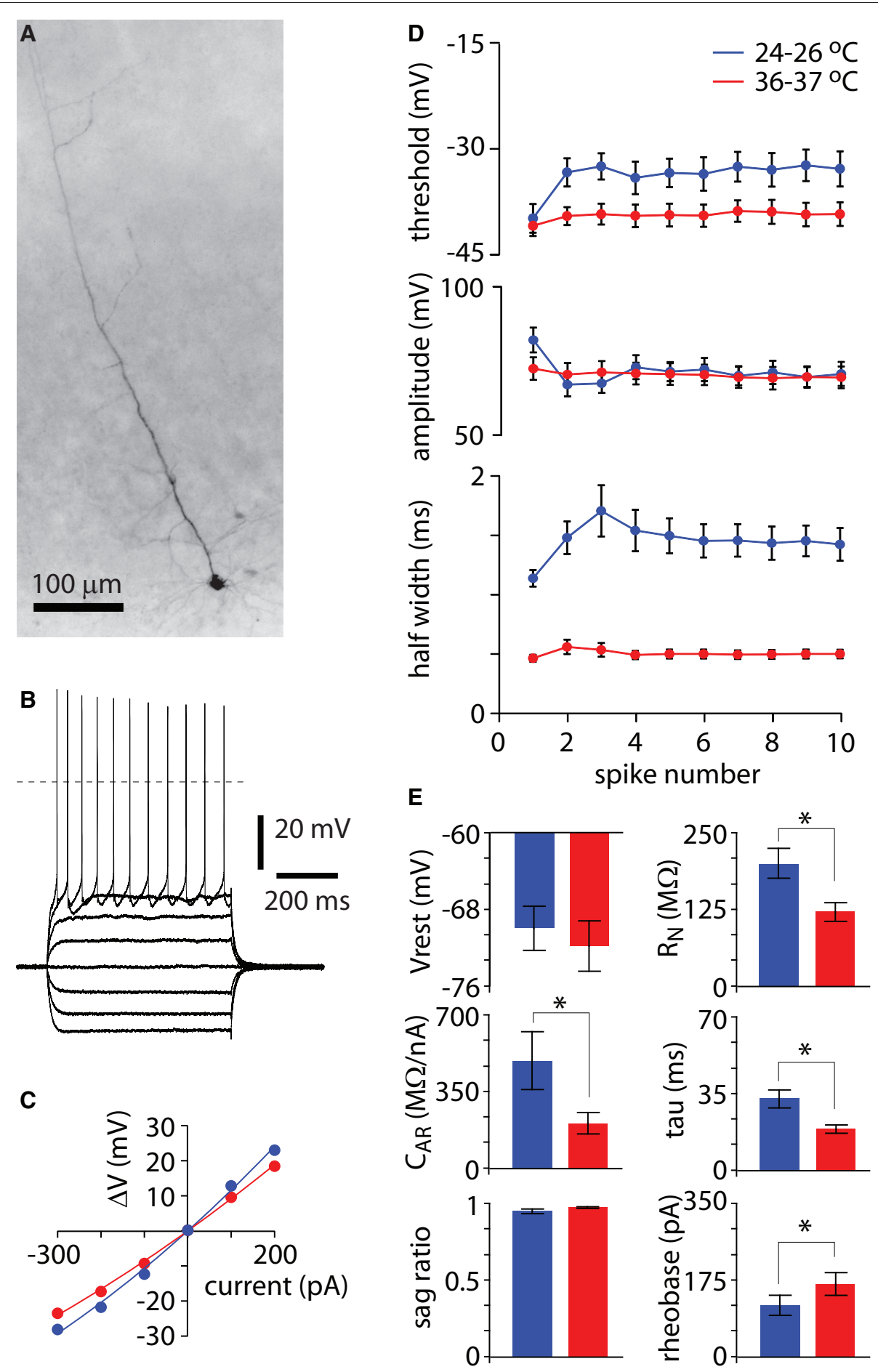

FIGURE 6 | Effect of temperature on spike waveform and membrane properties in layer 6 pyramidal neurons. (A) Widefield fluorescence image of a layer 6 pyramidal neuron, filled with Alexa 594 via the recording pipette. Note that the apical dendritic tree is intact. (B) Voltage responses to somatic current injection in the neuron in panel $\mathbf{A} .100 \mathrm{pA}$ current steps from -300 to $+400 \mathrm{pA}$ at $35^{\circ} \mathrm{C}$. (C) V-I curves at 26 (blue) and $35^{\circ} \mathrm{C}$ (red) for the neuron in panel $\mathbf{A}$, generated from results such as that in panel $\mathbf{B}$. Points denote steady-state voltage measurements

and curves are fits to $\Delta V=R_{N, 0} \Delta I+c_{A R} \Delta l^{2}$ (Waters and Helmchen, 2006). (D) Spike threshold, amplitude, and half width during $600 \mathrm{~ms}$ constant current injection at $36-37^{\circ} \mathrm{C}$ (red) and $24-26^{\circ} \mathrm{C}$ (blue) in 10 layer 6 pyramidal neurons. (E) Effects of temperature on resting membrane potential $\left(V_{\text {rest }}\right)$, input resistance $\left(R_{N}\right)$, anomalous

rectification $\left(C_{A R}\right)$, membrane time constant (tau), membrane sag, and rheobase. Each bar represents the mean \pm SEM from 10 neurons. * Indicates $P<0.05$. 


\section{APICAL DENDRITE IS REQUIRED FOR CHANGE IN SPIKING PATTERN WITH TEMPERATURE}

In layer 6 pyramidal neurons, the ISIs of spikes evoked by current injection into the apical dendrite are more variable than ISIs evoked by somatic current injection (Ledergerber and Larkum, 2010) and we have hypothesized that lower temperatures lead to irregular spiking by increasing the electrical coupling between the dendrites and soma (Hedrick and Waters, 2011). Hence we expect that temperature would have little or no effect on the spiking patterns of pyramidal neurons lacking an apical dendrite.

To test for temperature effects in neurons lacking an apical dendrite, we recorded from four layer 6 pyramidal neurons in slices cut at a slight angle to the major axis of the apical dendrites, such that the apical dendrites of many pyramidal neurons were cut at the surface of the slice, near the soma. In all four neurons we confirmed that the apical dendritic tree was largely absent by examining the morphology of the filled neuron by fluorescence microscopy (Figure 7A). In layer 6 neurons with truncated apical dendritic trees, changes in temperature had little or no effect on spiking pattern, which was consistent throughout the experiment (Figures 7B,C) and ISIs were similar at $24-26$ and $35-37^{\circ} \mathrm{C}$ (Figures 7D,E). These results support our hypothesis that the decrease in spiking regularity results from increased coupling of soma and apical dendrite with decreasing temperature.

\section{DISCUSSION}

Our results indicate that spiking pattern in both layer $2 / 3$ and layer 6 pyramidal neurons is variable, reflecting the degree to which the somatic and dendritic compartments are coupled. Both layer $2 / 3$ and layer 6 pyramidal neurons spike with more irregular patterns during constant current injection at $24-26^{\circ} \mathrm{C}$ than at physiological temperatures. Layer $2 / 3$ pyramidal neurons can adopt any of three spiking patterns at physiological temperatures: regular spiking, weak bursting, or adapting. Upon cooling the slice to $24-26^{\circ} \mathrm{C}$, these neurons tend to adopt adapting spiking patterns. Layer 6 pyramidal neurons can adopt any of four spiking patterns: regular spiking, weak bursting, adapting, or strong bursting. At physiological temperatures, layer 6 pyramidal neurons tend to adopt either regular spiking or weak bursting patterns, and at $24-26^{\circ} \mathrm{C}$ these neurons tend to adopt an adapting firing pattern. In both layer $2 / 3$ and layer 6 , ISIs were more variable at low temperatures than at physiological temperatures, with neurons that did not switch to a different spiking pattern displaying an increase in ISI variability at low temperature.

Lowering temperature to $24-26^{\circ} \mathrm{C}$ also affected action potential waveform and intrinsic membrane properties. In both layer $2 / 3$ and layer 6 pyramidal neurons, action potentials became broader upon cooling the slice to $24-26^{\circ} \mathrm{C}$. The threshold for the first action potential was unchanged, but threshold for spikes 2-10 was more depolarized at $24-26^{\circ} \mathrm{C}$ than at physiological temperatures.

\section{TEMPERATURE EFFECTS ON SPIKE WAVEFORM AND INTRINSIC MEMBRANE PROPERTIES}

In addition to affecting spiking pattern, a decline in temperature from $36-37^{\circ} \mathrm{C}$ to $24-26^{\circ} \mathrm{C}$ increased resting input resistance and rectification $\sim 200-300 \%$. The magnitude of this change is comparable to that observed in layer 5 pyramidal neurons and also in hippocampal pyramidal neurons ( Thompson et al., 1985; Shen and Schwartzkroin, 1988; Volgushev et al., 2000; Trevelyan and Jack, 2002; Lee et al., 2005; Hedrick and Waters, 2011). Hence these changes, that presumably underlie the change in electrical coupling of soma and dendrites, are common to many different types of pyramidal neuron.
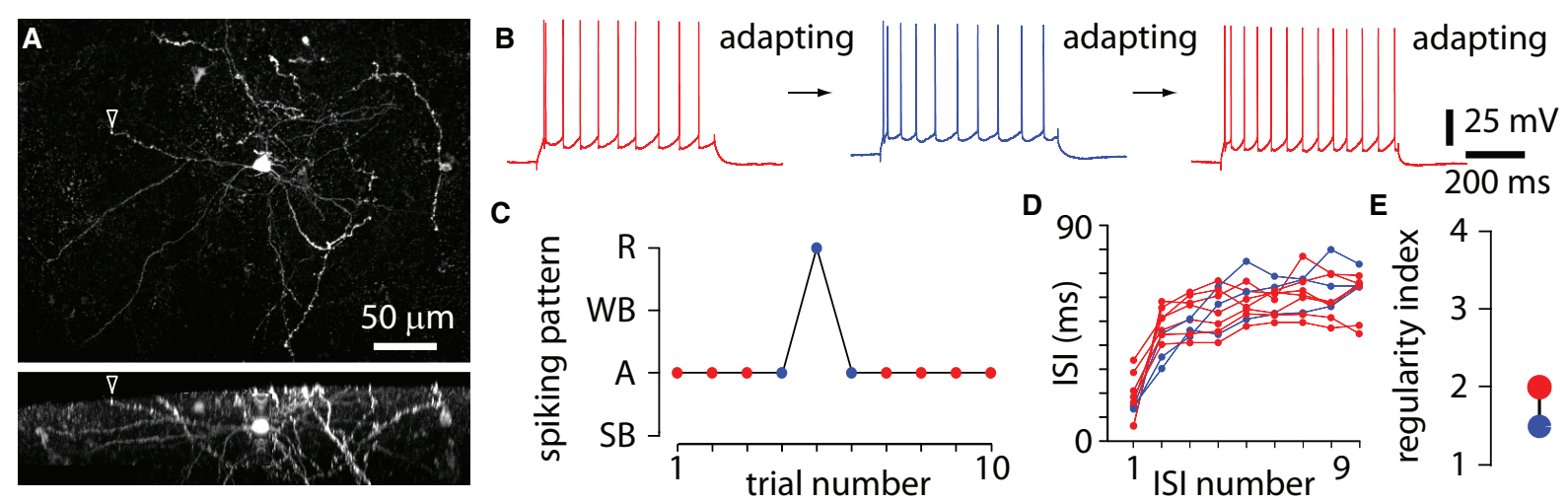

FIGURE 7 | Little temperature-sensitivity of the spiking pattern after loss of apical dendrite. (A) Maximum intensity projections from 2-photon $z$-stacks, showing a layer 6 pyramidal neuron which is missing much of its apical dendritic tree. The apical dendrite is severed at the surface of the slice (arrowhead). Lower panel shows the same neuron from the side, with the cut branch extending from the soma and rising toward the surface of the slice, where it is cut (arrowhead). (B) Voltage recordings from a layer 6 pyramidal neuron with a severed apical dendrite, showing spiking patterns in a series of trials, during which temperature was changed from $35-37^{\circ} \mathrm{C}$ (red) to $24-26^{\circ} \mathrm{C}$ (blue) and back to $35-37^{\circ} \mathrm{C}$. The spiking pattern of the neuron remained constant. (C) Spiking patterns for the neuron in panel B. The spiking pattern remained adapting at both temperatures, with only one trial displaying regular spiking. (D) The first nine inter-spike intervals (ISIS) for the 10 trials in panel $\mathbf{C}$. Note the lack of change in ISIs with temperature (in contrast with that in Figure 3). (E) Summary plot showing little change in spiking pattern in layer 6 pyramidal neurons with decreasing temperature. Blue dots and red dots represent average index of regularity at room temperature and physiological temperature, respectively, for four neurons. 


\section{COMMON EFFECT OF TEMPERATURE ON SPIKING PATTERNS IN LAYER 2/3, 5, AND 6 PYRAMIDAL NEURONS}

In an earlier study we hypothesized that lower temperatures lead to irregular spiking by increasing the electrical coupling between the dendrite and soma of layer 5 pyramidal neurons (Hedrick and Waters, 2011). This hypothesis resulted from two observations: (1) the input resistance of layer 5 pyramidal neurons increases with decreasing temperature; and (2) layer 5 pyramidal neurons adopt regular spiking patterns at physiological temperatures and burst at lower temperatures. Bursting in layer 5 pyramidal neurons results from the activation of sodium and calcium conductances in the apical dendrite (Williams and Stuart, 1999). Hence by increasing input resistance, lower temperatures increase the length constants of the dendrites, enhancing activation of dendritic conductances during somatic current injection, and the result is burst spiking patterns.

Here we have observed similar effects in layer $2 / 3$ and layer 6 pyramidal neurons, including an increase in input resistance and less regular spiking with decreasing temperature. Sodium and calcium conductances are present in the apical dendrites of layer 2/3 and layer 6 pyramidal neurons (Larkum et al., 2007; Ledergerber and Larkum, 2010) and in layer 6, activation of these conductances by dendritic current injection at physiological temperatures results in ISIs that are more variable than when spiking is evoked by somatic current injection (Ledergerber and Larkum, 2010). Hence the decrease in the regularity of spiking with declining temperature, as observed here, is consistent with the suggestion that temperature affects spiking pattern by affecting the electrical coupling between soma and apical dendrite.

If the mechanism by which temperature affects spiking pattern is similar in layer $2 / 3$, layer 5 , and layer 6 pyramidal neurons, why do layer $2 / 3$ and layer 6 pyramidal neurons typically not adopt burst spiking patterns at $24-26^{\circ} \mathrm{C}$ ? Dendritic spikes in layer $2 / 3$ and layer 6 pyramidal neurons, evoked by dendritic current injection, are briefer than in later 5 pyramidal neurons and do

\section{REFERENCES}

Agmon, A., and Connors, B. W. (1989). Repetitive burst-firing neurons in the deep layers of mouse somatosensory cortex. Neurosci. Lett. 99, 137-141.

Agmon, A., and Connors, B. W. (1992). Correlation between intrinsic firing patterns and thalamocortical synaptic responses of neurons in mouse barrel cortex. J. Neurosci. 12, 319-329.

Chagnac-Amitai, Y., Luhmann, H. J., and Prince, D. A. (1990). Burst generating and regular spiking layer 5 pyramidal neurons of rat neocortex have different morphological features. J. Comp. Neurol. 296, 598-613.

Cho, R. H., Segawa, S., Mizuno, A., and Kaneko, T. (2004). Intracellularly labeled pyramidal neurons in the cortical areas projecting to the spinal cord. I. Electrophysiological properties of pyramidal neurons. Neurosci. Res. 50, 381-394.

Connors, B. W., and Gutnick, M. J. (1990). Intrinsic firing patterns of diverse neocortical neurons. Trends Neurosci. 13, 99-104.

Connors, B. W., Gutnick, M. J., and Prince, D. A. (1982). Electrophysiological properties of neocortical neurons in vitro. J. Neurophysiol. 48, 1302-1320.

Dégenètais, E., Thierry, A. M., Glowinski, J., and Gioanni, Y. (2002). Electrophysiological properties of pyramidal neurons in the rat prefrontal cortex: an in vivo intracellular recording study. Cereb. Cortex 12, 1-16.

Hedrick, T., and Waters, J. (2011). Spiking patterns of neocortical L5 pyramidal neurons in vitro change with temperature. Front. Cell. Neurosci. 5:1. doi: 10.3389/fncel. 2011.00001

not depolarize the neuron long enough to support a burst of spikes (Schiller et al., 1997; Larkum et al., 2001, 2007; Ledergerber and Larkum, 2010). Hence decreasing temperature results in a decrease in the regularity of spiking at lower temperatures in all three types of pyramidal neuron, but with slightly different outcomes which are dictated by their dendritic conductances.

In vivo, spiking will be driven by synaptic activity, generally arriving in the dendritic tree, and is therefore, unlikely to replicate the patterns described here during constant current injection at the soma. Nonetheless our conclusion, that electrical coupling between soma and dendrite affect the spiking of pyramidal neurons, is likely to apply in vivo, where ongoing synaptic activity may dynamically alter the electrical properties of the dendritic tree and thereby the coupling between soma and dendrite. The idea that synaptic input to the proximal apical dendrites of layer 5 pyramidal neurons may functionally couple/decouple somatic and dendritic compartments has been suggested (Larkum et al., 2001). Our results suggest that ongoing synaptic activity might similarly couple and decouple the dendrites and thereby alter the spiking patterns of layer $2 / 3$ and layer 6 pyramidal neurons.

The idea that pyramidal neurons can change their spiking patterns is not new. Layer 5 pyramidal neurons can switch between regular and burst spiking patterns, not only with temperature (Hedrick and Waters, 2011), but probably also with ongoing synaptic activity (Steriade, 2004). Our results extend this idea to layer $2 / 3$ and layer 6 pyramidal neurons. Although often described as regular spiking neurons, the spiking patterns of layer $2 / 3$ and layer 6 pyramidal neurons are not fixed may vary considerably with prevailing conditions.

\section{ACKNOWLEDGMENTS}

We thank Becky Imhoff and Lauren Sybert for technical assistance. This work was supported by the National Institute of Mental Health (5T32MH067564-08 and 5R21MH085117-02) and the Brain Research Foundation (BRF SG 2011-018).

Larkum, M. E., and Zhu, J. J. (2002). Signaling of layer 1 and whiskerevoked $\mathrm{Ca}^{2+}$ and $\mathrm{Na}^{+}$action potentials in distal and terminal dendrites of rat neocortical pyramidal neurons in vitro and in vivo. J. Neurosci. 22, 6991-7005.

Larkum, M. E., Waters, J., Sakmann, B., and Helmchen, F. (2007). Dendritic spikes in apical dendrites of neocortical layer 2/3 pyramidal neurons. J. Neurosci. 27, 8999-9008.

Larkum, M. E., Zhu, J. J., and Sakmann, B. (2001). Dendritic mechanisms underlying the coupling of the dendritic with the axonal action potential initiation zone of adult rat layer 5 pyramidal neurons. J. Physiol. 533, 447-466.

Ledergerber, D., and Larkum, M. E. (2010). Properties of layer 6 pyramidal neuron apical dendrites. J. Neurosci. 30, 13031-13044.

Lee, J. C., Callaway, J. C., and Foehring, R. C. (2005). Effects of temperature on calcium transients and $\mathrm{Ca}^{2+}$ dependent afterhyperpolarizations in neocortical pyramidal neurons. J. Neurophysiol. 93, 2012-2020.

Mason, A., and Larkman, A. (1990). Correlations between morphology and electrophysiology of pyramidal neurons in slices of rat visual cortex II. Electrophysiology. J. Neurosci. 70, 1415-1428.

McCormick, D. A., Connors, B. W., Lighthall, J. W., and Prince, D. A. (1985). Comparative electrophysiology of pyramidal and sparsely spiny stellate neurons of the neocortex. J. Neurophysiol. 54, 782-806.

Nuñez, A., Amzica, F., and Steriade, M. (1993). Electrophysiology of cat association cortical cells in vivo: intrinsic properties and synaptic responses. J. Neurophysiol. 70, 418-430.

Schiller, J., Schiller, Y., Stuart, G., and Sakmann, B. (1997). Calcium action potentials restricted to distal 
apical dendrites of rat neocortical pyramidal neurons. J. Physiol. 505, 605-616.

Schwindt, P., and Crill, W. (1999). Mechanisms underlying burst and regular spiking evoked by dendritic depolarization in layer 5 cortical pyramidal neurons. J. Neurophysiol. 81, 1341-1354.

Shen, K. F., and Schwartzkroin, P. A. (1988). Effects of temperature alterations on population and cellular activities in hippocampal slices from mature and immature rabbit. Brain Res. 475, 305-316.

Silva, L. R., Amitai, Y., and Connors, B. W. (1991). Intrinsic oscillations of neocortex generated by layer 5 pyramidal neurons. Science 251, 432-435.
Steriade, M. (2004). Neocortical cell classes are flexible entities. Nat. Rev. Neurosci. 5, 121-134.

Thompson, S., Masukawa, L., and Prince, D. (1985). Temperature dependence of intrinsic membrane properties and synaptic potentials in hippocampal CA1 neurons in vitro. J. Neurosci. 5, 817-824.

Trevelyan, A. J., and Jack, J. (2002). Detailed passive cable models of layer $2 / 3$ pyramidal cells in rat visual cortex at different temperatures. J. Physiol. 539, 623-636.

Volgushev, M., Vidyasagar, T. R., Chistiakova, M., and Eysel, U. T. (2000). Membrane properties and spike generation in rat visual cortical cells during reversible cooling. J. Physiol. 522, 59-76.
Waters, J., and Helmchen, F. (2006). Background synaptic activity is sparse in neocortex. J. Neurosci. 26, 8267-8277.

Waters, J., Larkum, M., Sakmann, B., and Helmchen, F. (2003). Supralinear Ca2+ influx into dendritic tufts of layer $2 / 3$ neocortical pyramidal neurons in vitro and in vivo. J. Neurosci. 23, 8558-8567.

Williams, S. R., and Stuart, G. J. (1999). Mechanisms and consequences of action potential burst firing in rat neocortical pyramidal neurons. J. Physiol. 521, 467-482.

Conflict of Interest Statement: The authors declare that the research was conducted in the absence of any commercial or financial relationships that could be construed as a potential conflict of interest.

Received: 25 January 2012; accepted: 23 April 2012; published online: 14 May 2012.

Citation: Hedrick T and Waters J (2012) Effect of temperature on spiking patterns of neocortical layer 2/3 and layer 6 pyramidal neurons. Front. Neural Circuits 6:28. doi: 10.3389/fncir.2012.00028

Copyright (c) 2012 Hedrick and Waters.

This is an open-access article distributed under the terms of the Creative Commons Attribution Non Commercial License, which permits non-commercial use, distribution, and reproduction in other forums, provided the original authors and source are credited. 